\title{
Methylation and schizophrenia
}

Dynamic changes in the levels of GABA ( $\gamma$-aminobutyric acid)-ergic marker proteins are observed during brain development and in psychiatric disease, but whether these changes occur at the level of transcription or translation, or are due to post-translational modifications remains unclear. A recent study uncovers an important epigenetic mechanism that controls the expression of genes involved in GABA neurotransmission during normal cortical development and schizophrenia.

The authors showed that during maturation of the human prefrontal cortex (PFC), which starts prenatally and continues into puberty, there is a progressive increase in the number of GABAergic mRNA transcripts, including glutamic acid decarboxylase 1 (GAD1), a key enzyme for GABA synthesis. These increases were associated with chromatin remodelling, as an increase in histone H3 Lys4 (H3K4) methylation at GABAergic gene loci was also observed over this period of development.

Akbarian and colleagues found similar results in the mouse cerebral cortex, and so used mice to investigate the mechanism by which the H3K4 methylation of GABAergic chromatin occurs. Of the multiple histone methyltransferases that are expressed in mammals, only mixed lineage leukaemia 1 (MLL1) is strongly expressed in the adult human PFC and the mouse cerebral cortex. Examination of samples of cerebral cortex taken from adult $\mathrm{Mll1}^{+/-}$mice revealed a decrease in $\mathrm{H} 3 \mathrm{~K} 4$ methylation at the promoters of GABAergic genes. However, as the levels of the corresponding
GABAergic mRNAs did not change, MLL1 might function downstream from the transcriptional process to regulate GABAergic gene expression.

Interestingly, significant decreases in the levels of GAD1 mRNA and $\mathrm{H} 3 \mathrm{~K} 4$ methylation were found in the PFC of female patients with schizophrenia, but not male patients. Similar reductions were found in patients (both female and male) that are biallelic for GAD1 single nucleotide polymorphisms (SNPs), which have been identified as risk factors for the disease. Finally, the authors also show that chronic administration of the atypical antipsychotic clozapine increased the levels of MLL1 mRNA and GAD1 H3K4 methylation in mouse cerebral cortex, suggesting that the pharmacological profile of this drug extends beyond dopamine D2-like receptor antagonism.

Although it remains to be determined whether Mll1-deficient mice exhibit schizophrenia-associated behaviour, how SNPs affect GAD1 expression and why the effects appear to be gender-specific, these findings shed new light on the pathophysiology of schizophrenia and might open new avenues of research for the development of novel therapies.

\section{Monica Hoyos Flight}

\section{ORIGINAL RESEARCH PAPER}

Huang, H.-S. et al. Prefrontal dysfunction in schizophrenia involves mixed-lineage leukemia 1-regulated histone methylation at GABAergic gene promoters.J. Neurosci. 27, 11254-11262 (2007)

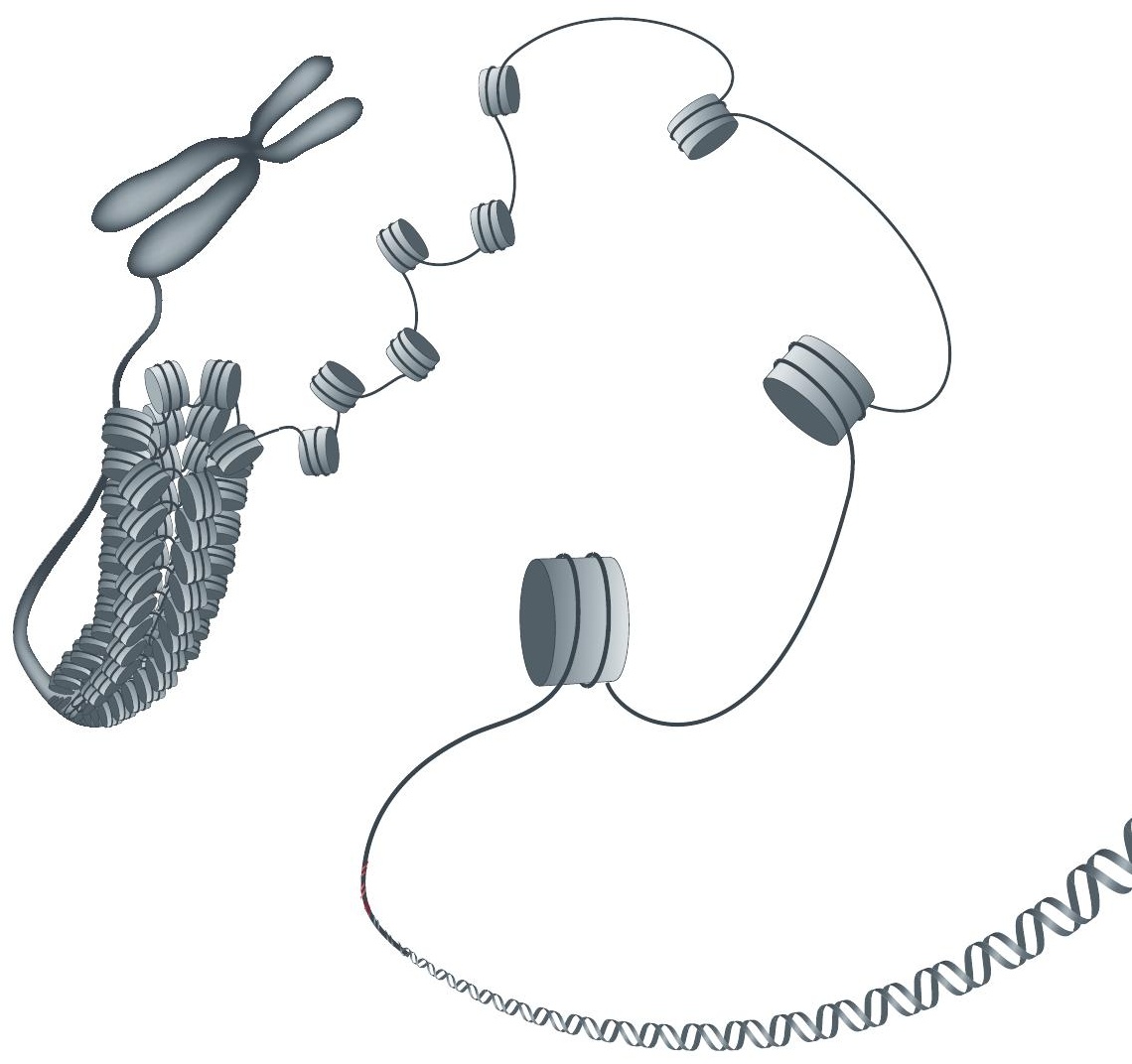

